\title{
Research of the Development of Multimedia Key Technology
}

\author{
Zhongsheng Wang ${ }^{1,}$ a and Xin Zhan ${ }^{1}$ \\ ${ }^{1}$ School of Computer Science and Engineering, Xi'an Technological University, Xi'an 710021, China \\ a59483672@qq.com
}

Keywords: Multimedia technology; Audio; Image; Video; Cartoon

\begin{abstract}
With the development of computer technology, multimedia technology is playing a important role in human's daily life and work based on computer technology, this Paper is starting from the history of multimedia technology, discussing the multimedia application, computer hardware and operation system supports multimedia technology, and elaborates multimedia technology development tendency.
\end{abstract}

\section{Introduction}

With the rapid development of computer technology, the computer-based technology of multimedia has been applied to variousaspects in society wildly.Multimedia technology is a technology which is the fast growing, most widely used and the fastest change in the modern information technology area, and also as a pot for development and competition in electronictechnique's area. Multimedia technology includes sounds, images, movie, video and other functions in one, with more and more popularity of high speed internet. Through the computer networking around the world and information resources sharing, it is widely used in industry, agriculture, services, education, and communication, military, financial and other business. In today which is high-speed development of modern technology, it has become a key to push society develop what is information transmission, processing and sharing.

\section{The History of Multimedia Technical Development}

Multimedia technology is an information technology which use interactive integrated technology and digital communication network technology to deal with some media-text, graphics, images, film, video and audio - and make information to build logical connection, as an interactive system to complete some interactive operations. It mainly relates to the multimedia's data processing technology — image processing, audio processing, video processing, etc., multimedia communication and distributed multimedia, multimedia applications, and other some respects. The United States apple was put forward the bitmap concept and mark the multimedia technology was born, in 1984.Then, the United States Commdore company put to market the first multimedia system - Amiga - all over the world, and it has a complete audio and video processing capabilities to make the world see the future what is multimedia technology.

There are three stages of the multimedia technology, includes: traditional media, streaming media and intelligent multimedia technology. The traditional media technology is the primary stage of multimedia, in this stage, all of the information has been processed after completely receiving. It has a low speed to process, and has a long time to process information, therefore, People have to spend much time to wait for the results. Streaming media is a new technology to solve the traditional multimedia problems, the so-called "streaming" is a meaner to transmit data. Using this technique, the computers have able to compute those have been received information when all of the information not arrive completely. This method is well solved the problem of multimedia information transmit on the network which is receiving and processing at the same time. In this way, if people want to receive the information, they don't have to wait a long time. And then receive at the same time - don't feel the file is not finished. Intelligent multimedia technology is a good 
combination in AI of some subjects and multimedia computer technology. Make full use of the ability which is the computer's fast operation, processing information-synthetically - such as sound, text and image. Using the method of interaction to offset the defect of computer intelligent, such as: the text of recognition and input, the language understanding and translation, the graphic recognition and understanding, robot vision and computer vision.

\section{Software Upgrades}

Multimedia software is a software system which is based on the multimedia hardware to design and develop.

The Development and Status Quo of Audio Processing Software. With the development of multimedia technology, audio processing software is also development. From Cool Edit Pro to Adobe Audition to Cubase, their ability to process strengthened, continuously.

Cool Edit Pro is the Syntrillium Software Corporation Company developed powerful Software in terms of multi-track recording and audio processing. It is also excellent software on digital music editor and MP3 production. Its amplification, reduce noise, compression, extension, echo, distortion, latency, and other functions. Cool Edit Pro V2.0 and Cool Edit Pro V2.1 are developed by the Cool Edit Pro, and they have more features than the Cool Edit Pro-128 rail audio, enhanced audio editing ability, more than 40 kinds of audio effects and etc. Adobe Audition is pushed by Adobe after the company purchase the Syntrillium Software Corporation and the software is designed for audio professionals who are radio equipment and post-production, therefore it can create high quality sound. The Major improvements that are compared with Cool Edit Pro as follows: support ASIO and VST plug-ins, mixing speed up more than three times, a new spectrum analyzer, etc. Cubase SX was pushed by Steinberg, it opened a new era in voice processing field. It added more than 70 new features - audio bending function, support external FX plugin, etc.

The above three software - although the Cool Edit is developed by the Audition, but the Audition is more easy to learn, In addition, Cubase is one of the most professional and more powerful software. The user can choose those software according to their needs.

The Development and Status Quo of Image Processing Software. Image processing technology is an emerging technology with the development of computer technology and VLSI in the 1960's. This section will elaborate the development of image processing software from Photoshop, ACDSee and CorelDRAW.

Photoshop is developed by Adobe, one of the most successful image processing software. From 1990 to 2012, the version is also from 1.0 to CS6. The function of Photoshop continuous enhancement - to improve the layer, increase the interpolation method and HUD features - make it used widely, have a friendly interface and can cross platform service. But it does not support large pixel image, and less on dealing with vector diagram. ACDSee is very popular software. From 1993 to 2012, the version is also from 1.0 to 15.0. With the improvement of the software-constantly-it realized the quick scan of the high pixel pictures, the third party plug-in extension and can support a variety of image formats - Gif, FPG, etc.. But it is just as a picture tool, cannot to support images of the secondary processing steadily and only use in Windows system. CorelDRAW is an image processing software which is Canada's Corel developed. It is a powerful software and widely used on mark design, Logo design, illustrations painted and some other fields. From 1989 to 2012, the version is also from 1.0 to X6, the software function is also growing. Until now, CorelDRAWX6 is the most powerful version. This software has those advantages what is has rich color system, support various color, support the background and save functions, support for vector image processing functions. However, the software is too big so that need a lot of resources to support when it is working.

The three image processing software has its own characteristics: ACDSee can quickly open and browse the images, but the function for image processing is not strong. PhotoShop focus on image 
processing, can't deal with vector graphics. CorelDRAW focus on graphics. Users should according to the demand to choose software.

The Development and Status Quo of Video Processing Software. Video processing is a technology which is refers to make the dynamic image become static — catch, Record, store, transfer, and output. This section will introduce those two video processing software-Corel Video Studio Pro and Premiere.

Corel Video Studio Pro is a powerful video editing software, it can capture the image and edit the image. Such as transform MV, DV, V8, TV and Real-time capture the picture. Now, it has updated to Corel Video Studio Pro X7 version. Added some function-Screen recording, HTML5 optimization, Cascade rail, etc. The Premiere was pushed by Adobe. It is a good software for edit and compatibility, and are widely used in advertising and television production. The latest version is Adobe Premiere CC2014, it is increased the multi-core GPU support, links, positioning function closed caption function, etc.

This software is easy to use, and suitable for family use. Premiere have a powerful function, it is a necessary tools for professional. If you still want more, you can try this software that is Sony Vegas.

The Development and Status Quo of Dynamic Picture Processing Software. The dynamic picture is made up by Continuous but not the same picture. It divided into 2D and 3D. This section introduces the 2D software is Flash, 3D software is 3DS MAX.

Flash is a two-dimensional vector animation software which is designed by Macromedia company, and later acquired by Adobe. And they updated software version constantly.

From 1995 to 2013, the version is also update to Flash Professional CC, the software function is also growing and was popular of users. The main function of this software is that synchronization, support 64 - bit architecture, real-time drawing and real-time color preview, etc. 3DS MAX is a 3Dedit software by Auto Desk. From the beginning of 1997, it has pushed many versions. The latest version is Autodesk 3DS MAX 2013, it has bring users a higher production efficiency and unique visual modeling capabilities.

Flash is a very good vector animation software-based on the flow and vector control technology — and made animation delicately. And widely used in web animation design.3DS MAX has a powerful function, easy to extend, simple to operate. It is widely used in 3D modeling, animation, games, construction and other area.

Other Technical Development Status. This section is about some processing software - audio, Image, video, animation. Designer creates a multimedia authoring tool for what to combine software.

Multimedia authoring tools is a tool what is combined with text, static images, video, graphics, images, animation, sound, etc. processing. Also called for multimedia develop platform. Authorware is a multimedia authoring tools which pushed by Macromedia. It has such main features - object-oriented visualization programming, rich multimedia material, enhanced ability to scale and so on. This software can be used to make multimedia application system-multimedia teaching system, multimedia database, military command system and simulation training, etc.

\section{The Support of Computer Hardware for Multimedia Technology}

Multimedia software can be able to process sound, images, video and so on, computer hardware plays an important role in multimedia technology. Computer hardware is as follows: the CPU, memory, audio card, the graphics accelerator card, video card, scan card and so on.

With the development of the central processing unit (CPU), multimedia CPU generates through it. Multimedia CPU is with CPU of MMX technology, MMX technology is a kind of multimedia extension structure, it greatly improved the function of the computer application in multimedia and communication way. Multimedia CPU is suitable fora lot of the processing of graphics and image 
data, and improves the speed of MPEG video, music synthesis, speech recognition and son on, whose target is 3D graphics, pictures, motion image. Memory is a kind of equipment to stores procedure and various data, which is divided into internal memory and external memory. The capacity of internal memory is $8 \mathrm{~GB}$, the capacity of external memory is a few $\mathrm{T}$, which can satisfy the storage of graphics, sound, video. Audio card is the most basic part of the multimedia technology, it is one of the transformation from acoustic digital signal hardware. The basic function of audio card is to switch the original sound from the microphone, CD signal , output to the headphones, speakers, amplifiers, tape recorders and other sound equipment, or to make musical sounds wonderful music equipment through a digital interface, realizing the collection of sound, achieve sound processing after voice processing software to output. Scan card is used to connect all kinds of graphics scanners, commonly used static pictures, text and other input devices, obtaining material pretreatment, input material through image processing software for processing, we can use image processing software to process input material, need high performance equipment, so add graphics accelerator card to realize the graphic processing. Video card is to connect the camera, VCR, TV and other equipment, complete the acquisition, processing, and performance of various animation and digital video media.

\section{The Support of Development of the Operating System on Multimedia Technology}

The operating system is the control center of computer software and hardware resources, organizes and calls the hardware and software resources rationally, accomplish the tasks that need to be done. Multimedia operating system supports multimedia real-time application, the first priority is to schedule all available resources to complete real-time control tasks, secondly to improve the efficiency of the use of a computer system. The main problems to solve in multimedia processing include interruption waiting, real-time scheduling, time limit and recovery management and resource management based on QoS. In order to solve the main problem, experts developed a new multimedia operating system architecture. Interrupt to wait, preempt the real-time operating system is based on task priority, kernel can preempt running task CPU use, and give use to the ready state priorities change task, namely the arrival of the higher priority task, the rest of the tasks that are running can be interrupted. Real-time scheduling, CPU can do real-time processing through efficient real-time scheduling, real-time task scheduler does the scheduling in order to meet the deadline. Real-time scheduling algorithms can be divided into three types: priority-based scheduling algorithm, scheduling algorithm based on CPU usage percentage of Shared, and a process scheduling algorithm based on time. QoS management can be divided into two kinds of control modes: static and dynamic. Static control mode refers to know a QoS level before the communication, and do the maintenance throughout the connection survival during the specified level. Dynamic control mode allows the user to survive in the whole connection during initial QoS value adjustment. And there are two kinds of gist to modify the QoS level, can be adjusted according to the system resources available, or according to the needs of the task.

\section{The Prospect of the Development to Multimedia Technology.}

From the perspective of the development trend of multimedia technology, there are two directions of the development of multimedia technology: one is the development trend of network; the other is components, intelligent and embedded of multimedia terminal.

The Development Trend of Multimedia Network. The development of multimedia technology can make the multimedia computer form a more perfect computer supported cooperative work environment, eliminate the obstacles of distance and the obstacles of time distance, provide better information service for human beings.

Interactive and dynamic multimedia technology can create a more vivid $2 \mathrm{D}$ and $3 \mathrm{D}$ scene in the network environment. 
The development of the multimedia interactive technology, the multimedia technology, uses a variety of sensory channel and motion channel, through the data glove and track sign language information, to extract the speaker-dependent facial features, synthetic facial movements and facial expressions, in parallel, and a very precise way to interact with the computer system on the basis of the pattern recognition, holographic images, natural language understanding and new sensor technology. It can improve the naturalness and the efficiency of the human-computer interaction, realize the realistic output marked in 3D virtual reality.

The Trend of Multimedia Technology of Components, Intelligence and Embedding. Now, the structure of multimedia computer hardware, the interface of video and audio software is improved continuously. Especially, using the plan which is architecture design, algorithm and software can improve computer's power. Thus we want to meet the need of multimedia network environment, to develop and research the multimedia software to make it more intelligent.

Multimedia system can be used in all aspects of people's life and work - in the area of industrial control and business management, medical electronic equipment, multimedia mobile phone, PDA, hand navigator, entertainment and other area.

\section{Conclusions}

This paper introduces the development of computer technology and software of audio processing, video processing, image processing, etc. Computer hardware and operating system support for multimedia technology. Finally tell the trend of the development of multimedia technology. It has some significance for the research of multimedia technology.

\section{References}

[1] Huang Chunhua. A brief discussion on the development prospect of multimedia computer technology [A].Science and Technology Innovation Herald, 2010.

[2] Wang Zhongsheng, Ma Jing. Multimedia technology application foundation. Tsinghua University Press, 2012.

[3] Lu Guanming, Cheng Xiaogang Qin Lei, etc. Audio processing technology in the production of multimedia courseware. Practical Electronics, 2014

[4] Lin Jian, Liu smiled. Introduction and use of several audio post processing software. Television Caption Effects Animation, 2009.

[5] Zou Jinchi, Bao Jingfu, Sun Benjin. The image software design and application. University of Electronic Science and Technology, 2013.

[6] He Xiaoqin, Peng Xiaoxia. Photoshop CS6: From entry to master. Tsinghua University Press, 2014

[7] Wang Yinghua, Huang Wei. Premiere Pro CS6. Tsinghua University Press, 2014.

[8] Cheng Yaqian, Zhang Jinping. Analysis of contemporary digital animation cutting-edge technology. Shandong Normal University, 2010.

[9] Li Lianghui. Flash CS6: Introductory and advanced. Tsinghua University Press, 2014. 